Doi: HTTPS://DOI.ORG/10.23910/2/2020.0373

\title{
Efficacy of Fungicidal Seed Treatments on Growth and Yield Parameters of Green Gram
}

\author{
A. J. Deshmukh ${ }^{1 *}$, A. N. Sabalpara ${ }^{2}$ and V. A. Patil ${ }^{2}$
}

${ }^{1}$ Navsari Agricultural University, College of Agriculture, Waghai, Gujarat (394 730), India

${ }^{2}$ Navsari Agricultural University, Navsari, Gujarat (396 450), India

\section{Corresponding Author}

A. J. Deshmukh

e-mail: amol_deshmukhnau@nau.in

\author{
Article History \\ Article ID: IJEP0373 \\ Received in $02^{\text {nd }}$ April, 2020 \\ Received in revised form $19^{\text {th }}$ April, 2020 \\ Accepted in final form $25^{\text {th }}$ April, 2020
}

\begin{abstract}
The experiment was conducted to perceive the effect of fungicidal seed treatment on growth and yield parameters of green gram cv. GM-3 with nine different fungicides. The results revealed that dry seed treatment with either mix formulation of carbendazim+mancozeb or thiophanate methyl or carbendazim @ $2.5 \mathrm{~g} \mathrm{~kg}^{-1}$ seeds is very effective in field to get maximum seed germination, better plant growth, root growth, yield parameters and yield. Seed germination was maximum in dry seed treatment with carbendazim + mancozeb (83.85\%) which was statistically at par with thiophanate methyl (83.58\%), carbendazim (82.53\%) and captan (80.77\%). Plant height was maximum in dry seed treatment in mixed formulation carbendazim+mancozeb $(41.53 \mathrm{~cm})$ which was statistically at par with thiophanate methyl $(39.91 \mathrm{~cm})$, carbendazim $(39.67 \mathrm{~cm})$ and captan $(37.00 \mathrm{~cm})$. Average numbers of pods plant ${ }^{-1}$ were maximum in dry seed treatment with carbendazim+mancozeb (12.33) which was statistically at par with thiophanate methyl (12.17), carbendazim (11.90), captan (11.85), thiram (11.58) and difenconazole (11.09). Average numbers of seeds pod ${ }^{-1}$ was maximum in dry seed treatment with carbendazim+mancozeb (7.58) which was statistically at par with thiophanate methyl (7.48), carbendazim (7.31), captan (7.28) and thiram (7.12). The pooled data of two years indicated that grain yield was significantly higher in dry seed treatment with carbendazim + mancozeb $\left(818.40 \mathrm{~kg} \mathrm{ha}^{-1}\right)$ as compared to the rest but was statistically at par with thiophanate methyl $\left(790.73 \mathrm{~kg} \mathrm{ha}^{-1}\right)$ and carbendazim $\left(771.35 \mathrm{~kg} \mathrm{ha}^{-1}\right)$ followed by all the other treatments.
\end{abstract}

Keywords: Fungicides, green gram, growth, seed treatment, yield

\section{Introduction}

Green gram [Vigna radiata (L.) Wilczek] is an important pulse crop (John, 1991). Disease is the major constraint in economic crop production as they inflict heavy losses. Like other crops, green gram is also attacked by many diseases during seed germination to seed production and maturity. Over 35 fungal pathogens, few viral, bacterial and nematode species are known to attack green gram resulting into substantial yield losses (Agrawal, 1989). Among these, seed borne fungal diseases are important in reducing the yield and seed quality of green gram (Sinha and Prasad, 1981). Most of the fungal diseases such as anthracnose, leaf spots, leaf blights and root rot causing severe losses are seed borne in nature in green gram. Hence, seeds of moong have been reported to play an important role in the dissemination of various pathogens (Rangaswami and Prasad, 1960; Rayen, 1961). These fungi affect developing seeds adversely such as seed discoloration, molding of seed surface, endosperm degradation and reduced seed filling resulting in seed deformities such as shriveling of seeds as well as small seed formation. The seed infecting fungi not only damages the quantity but also quality of seed.
Similarly, nutritional status of green gram seeds is likely to be hampered by depleting the protein content and by the disturbance of the reducing sugar as well as starch content due to seed mycoflora (Bilgrami et al., 1978). To increase the production of green gram qualitatively and quantitatively, farmer requires healthy quality seeds with high percentage of germination and purity. Hence, it is imperative that the seeds must be tested before they are sown in the field. Another adverse effect of seed borne pathogens is that it will contaminate the areas which were disease free previously. Hence, it necessitates the eradication of seed borne inoculum through various seed treatments and through the enforcement of proper domestic and international quarantine acts and procedures. Degree of damage to the green gram seeds is influenced by various biotic and abiotic factors along with virulent pathogen, varietal susceptibility and prevailing humidity particularly during developing pod-stage and harvesting time etc. Seed treatment is the oldest practice in plant protection and now, this is an attractive delivery system with either fungicide of bioprotectants. Fungicides applied to seeds may not only protect seeds from seed borne diseases but also protect roots and increase the plant growth indirectly. 
Hence, the present investigation was conducted to perceive the effect of fungicidal seed treatment on growth and yield parameters of green gram with nine different fungicides in green gram cv. GM-3.

\section{Materials and Methods}

A field trial was conducted for two seasons at N.M. College of Agriculture, Research Farm, N.A.U., Navsari. Agro climatic sub region was South Gujarat heavy rainfall zone, variety was GM3. Seeds collected from Pulse Research Station, N.A.U., Navsari were taken and sown after dry seed treatment with different fungicides viz., Carbendazim $\left(2.5 \mathrm{~g} \mathrm{~kg}^{-1}\right)$, Kasugomycin $(2.5 \mathrm{~g}$ $\left.\mathrm{kg}^{-1}\right)$, Thiram $\left(3.5 \mathrm{~g} \mathrm{~kg}^{-1}\right)$, Captan $\left(3.5 \mathrm{~g} \mathrm{~kg}^{-1}\right)$, Carbendazim + mancozeb (2.5 $\left.\mathrm{g} \mathrm{kg}^{-1}\right), \operatorname{MEMC}\left(2.5 \mathrm{~g} \mathrm{~kg}^{-1}\right)$, Mancozeb $\left(3.5 \mathrm{~g} \mathrm{~kg}^{-1}\right)$, Thiophanate methyl $\left(3.5 \mathrm{~g} \mathrm{~kg}^{-1}\right)$ and Difenconazole $\left(2.5 \mathrm{~g} \mathrm{~kg}^{-1}\right)$. Recommended agronomic practices like seed rate, spacing. Irrigation, fertilizer application and intercultural operations were followed during whole experiment.

\subsection{Pre emergence mortality, post emergence mortality and seed germination}

The observations on the pre-emergence mortality were taken by observing the germinated and total sown seeds in each row in a plot. The possibility of the cause of the preemergence mortality due to seed infecting fungi in respective treatment was confirmed by observing the un-germinated/ rotted seeds after digging out from the plots through naked eye or microscopic observations. The percentage of diseased un-germinated seeds were recorded at 10 days of sowing and pre-emergence mortality (\%) was calculated by using the formula:

Pre emergence mortality $=$ (No. of diseased un-germinated seeds / plot) / (Number of sown seeds/ plot) x100

The observations on the post-emergence mortality were taken by observing the numbers of rotted or damping off of seedlings at 10 days of sowing. The numbers of died seedlings recorded during observations were used to calculate post-emergence mortality. It was calculated by using the formula:

Post emergence mortality $=$ No. of diseased died seedling / plot) / (Number of sown seeds/ plot) x 100

Total numbers of normal seedlings/plot was recorded at 10 days of sowing and germination was calculated as by using the formula:

Seed germination $(\%)=$ (Number of normal seedlings / pot) / (Number of total plants/ plot) $\times 100$

\subsection{Growth parameters}

The observations on growth parameters were recorded at 45 days after sowing by selecting 10 plants per plot randomly in first year with standard protocol.

i. Shoot length/ plant height $(\mathrm{cm})$ : Shoot lengths of randomly selected 10 plants were measured from ground level to the growing apex. ii. Main root length $(\mathrm{cm})$ : Main root length of randomly selected 10 plants was taken from ground level to the root apex by uprooting the plants by giving light watering followed by digging the rhizosphere carefully without disturbing the root system. After uprooting of the plants, the roots were washed carefully with water to remove the soil which was attached on it.

iii. Number of root nodules: Observations on number of effective root nodules/plant was also taken from the uprooted plants.

\subsection{Yield and yield parameters}

The observations on yield and yield parameters were taken at the time of harvest.

i. Number of pods per plant: Number of pods per plant was recorded from 10 randomly selected plants at harvest in the first year.

ii. Number of seeds per pod: Twenty five pods were selected randomly from the bulk harvest of 10 selected plants. Average numbers of seeds per pod were recorded after manual splitting of the pods in the first year.

iii. Test weight (1000 seeds): Thousand dry seeds were randomly taken from the harvested bulk of each plot. These seeds were then weighed on microbalance for recording test weight in the first year.

iv. Yield: The seed yield per plot was recorded after weighing the seeds of the net plot. The yield per hectare was calculated on the basis of yield per net plot at the experiment in both the year.

\section{Results and Discussion}

\subsection{Pre emergence mortality}

The pooled data of both the years indicated that preemergence mortality was significantly reduced in all the treatments over the control. It was significantly lower in dry seed treatment with carbendazim + mancozeb (9.67\%) as compared to the rest but was statistically at par with thiophanate methyl (9.70\%) and carbendazim (10.45\%). Next best in order to merit was captan (11.48\%) followed by thiram (12.78\%). Pre-emergence mortality was comparatively higher in difenconazole (14.18\%), MEMC (15.10\%), mancozeb $(15.35 \%)$ and kasugamycin (15.39\%). The highest preemergence mortality was recorded in the control $(21.50 \%)$.

\subsection{Post emergence mortality}

The pooled data of both the years indicated that the per cent post-emergence mortality was significantly reduced in all the treatments over the control. It was significantly lower in dry seed treatment with carbendazim + mancozeb (6.48\%) as compared to the rest but was statistically at par with thiophanate methyl (6.72\%) and carbendazim (7.02\%). Next best in order to merit was captan, (7.75\%) followed by thiram (8.85\%). Comparatively higher post-emergence 
mortality was found in difenconazole $(9.42 \%)$ followed by MEMC (9.48\%), mancozeb (9.52\%) and kasugamycin (9.84\%). The highest post-emergence mortality was recorded in the control (11.62\%).

\subsection{Seed germination}

The pooled data of both the years indicated that the per cent seed germination was significantly increased in all the treatments over the control. It was maximum in dry seed treatment with carbendazim + mancozeb $(83.85 \%)$ and was significantly superior over the rest but was statistically at par with thiophanate methyl (83.58\%), carbendazim (82.53\%) and captan $(80.77 \%)$. Next best in order to merit was thiram (78.37\%) followed by difenconazole (76.40\%), MEMC (75.42\%), mancozeb (76.12\%) and kasugamycin $(74.77 \%)$. The lowest seed germination was found in the control (66.88\%). Maximum increase in seed germination was recorded in carbendazim + mancozeb $(25.37 \%)$ followed by thiophanate methyl (24.97\%), carbendazim (23.40\%), captan (20.77\%), thiram (17.18\%), difenconazole (76.40\%), MEMC (12.77\%), mancozeb (12.32\%) and kasugamycin (11.80\%).

\subsection{Growth parameters}

Results obtained on different growth parameters in the field study are presented in Table 1 to 3 .

\subsubsection{Plant height}

Average plant height was significantly increased in all the treatments over the control. It was maximum in dry seed treatment with carbendazim + mancozeb $(41.53 \mathrm{~cm})$ which was statistically at par with thiophanate methyl $(39.91 \mathrm{~cm})$, carbendazim $(39.67 \mathrm{~cm})$ and captan $(37.00 \mathrm{~cm})$. Next best treatment in order to merit was thiram $(35.89 \mathrm{~cm})$ followed by difenconazole $(35.73 \mathrm{~cm}), \operatorname{MEMC}(33.55 \mathrm{~cm})$, mancozeb $(32.66 \mathrm{~cm})$ and kasugamycin $(31.67 \mathrm{~cm})$. Plant height was recorded lowest in the control $(28.23 \mathrm{~cm})$. Maximum increase in plant height was recorded in carbendazim + mancozeb (47.11\%) followed by thiophanate methyl (41.37\%),

\begin{tabular}{|c|c|c|c|c|c|c|c|c|c|c|c|}
\hline \multirow[t]{2}{*}{$\begin{array}{l}\text { Sr. } \\
\text { No. }\end{array}$} & \multirow[t]{2}{*}{ Treatment } & \multicolumn{3}{|c|}{$\begin{array}{l}\text { Av. pre-emergence mor- } \\
\text { tality (\%) }\end{array}$} & \multicolumn{3}{|c|}{$\begin{array}{l}\text { Av. post emergence mor- } \\
\text { tality (\%) }\end{array}$} & \multicolumn{4}{|c|}{ Av. seed germination (\%) } \\
\hline & & $\begin{array}{l}\text { First } \\
\text { year }\end{array}$ & $\begin{array}{l}\text { sec- } \\
\text { ond } \\
\text { year }\end{array}$ & Pooled & $\begin{array}{l}\text { First } \\
\text { year }\end{array}$ & $\begin{array}{l}\text { second } \\
\text { year }\end{array}$ & Pooled & $\begin{array}{l}\text { First } \\
\text { year }\end{array}$ & $\begin{array}{l}\text { second } \\
\text { year }\end{array}$ & Pooled & $\begin{array}{c}\text { In- } \\
\text { crease } \\
(\%)\end{array}$ \\
\hline 1. & $\begin{array}{l}\text { Carbendazim } \\
\text { (Bavistin 50\% WP) }\end{array}$ & $\begin{array}{l}18.91 \\
(10.5)\end{array}$ & $\begin{array}{c}18.80 \\
(10.39)\end{array}$ & $\begin{array}{l}18.86 \\
(10.45)\end{array}$ & $\begin{array}{l}15.38 \\
(7.17)\end{array}$ & $\begin{array}{l}15.06 \\
(6.88)\end{array}$ & $\begin{array}{l}15.22 \\
(7.02)\end{array}$ & $\begin{array}{c}65.27 \\
(82.33)\end{array}$ & $\begin{array}{c}65.58 \\
(82.73)\end{array}$ & $\begin{array}{c}65.43 \\
(82.53)\end{array}$ & 23.40 \\
\hline 2. & $\begin{array}{l}\text { Kasugomycin (Kasugo- } \\
\text { mycin } 12 \% \text { WP) }\end{array}$ & $\begin{array}{c}23.17 \\
(15.48)\end{array}$ & $\begin{array}{l}23.03 \\
(15.3)\end{array}$ & $\begin{array}{c}23.10 \\
(15.39)\end{array}$ & $\begin{array}{c}18.39 \\
(10.00)\end{array}$ & $\begin{array}{l}18.09 \\
(9.68)\end{array}$ & $\begin{array}{l}18.24 \\
(9.84)\end{array}$ & $\begin{array}{c}59.71 \\
(74.52)\end{array}$ & $\begin{array}{c}60.39 \\
(75.02)\end{array}$ & $\begin{array}{c}60.05 \\
(74.77)\end{array}$ & 11.80 \\
\hline 3. & $\begin{array}{l}\text { Thiram (Thirum 75\% } \\
\text { WP) }\end{array}$ & $\begin{array}{c}21.01 \\
(12.86)\end{array}$ & $\begin{array}{l}20.88 \\
(12.7)\end{array}$ & $\begin{array}{c}20.95 \\
(12.78)\end{array}$ & $\begin{array}{l}17.41 \\
(9.00)\end{array}$ & $\begin{array}{l}17.11 \\
(8.70)\end{array}$ & $\begin{array}{l}17.26 \\
(8.85)\end{array}$ & $\begin{array}{c}62.17 \\
(78.14)\end{array}$ & $\begin{array}{c}62.54 \\
(78.60)\end{array}$ & $\begin{array}{c}62.36 \\
(78.37)\end{array}$ & 17.18 \\
\hline 4. & $\begin{array}{l}\text { Captan (Captan 75\% } \\
\text { WP) }\end{array}$ & $\begin{array}{c}19.86 \\
(11.54)\end{array}$ & $\begin{array}{l}19.73 \\
(11.4)\end{array}$ & $\begin{array}{c}19.81 \\
(11.48)\end{array}$ & $\begin{array}{l}16.30 \\
(7.90)\end{array}$ & $\begin{array}{l}15.99 \\
(7.61)\end{array}$ & $\begin{array}{l}16.14 \\
(7.75)\end{array}$ & $\begin{array}{l}63.86 \\
(80.56)\end{array}$ & $\begin{array}{c}64.17 \\
(80.99)\end{array}$ & $\begin{array}{c}64.01 \\
(80.77)\end{array}$ & 20.77 \\
\hline 5. & $\begin{array}{l}\text { Carbendazim +manco- } \\
\text { zeb (Sixer } 75 \% \text { WP) }\end{array}$ & $\begin{array}{l}18.17 \\
(9.72)\end{array}$ & $\begin{array}{l}18.05 \\
(9.6)\end{array}$ & $\begin{array}{l}18.12 \\
(9.67)\end{array}$ & $\begin{array}{l}14.89 \\
(6.62)\end{array}$ & $\begin{array}{l}14.57 \\
(6.35)\end{array}$ & $\begin{array}{l}14.73 \\
(6.48)\end{array}$ & $\begin{array}{c}66.17 \\
(83.66)\end{array}$ & $\begin{array}{c}66.51 \\
(84.05)\end{array}$ & $\begin{array}{c}66.34 \\
(83.85)\end{array}$ & 25.37 \\
\hline 6. & MEMC (Emisan 6\% WP) & $\begin{array}{c}22.94 \\
(15.19)\end{array}$ & $\begin{array}{c}22.79 \\
(15.00)\end{array}$ & $\begin{array}{l}22.87 \\
(15.1)\end{array}$ & $\begin{array}{l}18.08 \\
(9.64)\end{array}$ & $\begin{array}{l}17.78 \\
(9.33)\end{array}$ & $\begin{array}{l}17.93 \\
(9.48)\end{array}$ & $\begin{array}{c}60.20 \\
(75.17)\end{array}$ & $\begin{array}{c}60.45 \\
(75.67)\end{array}$ & $\begin{array}{c}60.32 \\
(75.42)\end{array}$ & 12.77 \\
\hline 7. & $\begin{array}{l}\text { Mancozeb \(Diathane } \\
\text { M-45, 75\% WP) }\end{array}$ & $\begin{array}{c}23.15 \\
(15.45)\end{array}$ & $\begin{array}{c}22.99 \\
(15.26)\end{array}$ & $\begin{array}{c}23.07 \\
(15.36)\end{array}$ & $\begin{array}{l}18.06 \\
(9.68)\end{array}$ & $\begin{array}{l}17.75 \\
(9.37)\end{array}$ & $\begin{array}{l}17.91 \\
(9.52)\end{array}$ & $\begin{array}{c}60.29 \\
(74.87)\end{array}$ & $\begin{array}{c}60.27 \\
(75.37)\end{array}$ & $\begin{array}{c}60.28 \\
(75.12)\end{array}$ & 12.32 \\
\hline 8. & $\begin{array}{l}\text { Thiophanate methyl } \\
\text { (Topsin M- } 75 \% \text { WP) }\end{array}$ & $\begin{array}{l}18.20 \\
(9.76)\end{array}$ & $\begin{array}{l}18.09 \\
(9.64)\end{array}$ & $\begin{array}{l}18.15 \\
(9.7)\end{array}$ & $\begin{array}{l}15.16 \\
(6.86)\end{array}$ & $\begin{array}{l}14.83 \\
(6.58)\end{array}$ & $\begin{array}{l}14.99 \\
(6.72)\end{array}$ & $\begin{array}{c}65.97 \\
(83.38)\end{array}$ & $\begin{array}{c}66.39 \\
(83.78)\end{array}$ & $\begin{array}{c}66.18 \\
(83.58)\end{array}$ & 24.97 \\
\hline 9. & $\begin{array}{l}\text { Difenconazole } \\
\text { (Score } 25 \% \mathrm{EC} \text { ) }\end{array}$ & $\begin{array}{c}22.19 \\
(14.26)\end{array}$ & $\begin{array}{c}22.05 \\
(14.09)\end{array}$ & $\begin{array}{c}22.12 \\
(14.18)\end{array}$ & $\begin{array}{l}18.02 \\
(9.58)\end{array}$ & $\begin{array}{l}17.72 \\
(9.27)\end{array}$ & $\begin{array}{l}17.87 \\
(9.42)\end{array}$ & $\begin{array}{c}60.77 \\
(76.16)\end{array}$ & $\begin{array}{c}61.10 \\
(76.64)\end{array}$ & $\begin{array}{c}60.94 \\
(76.40)\end{array}$ & 14.23 \\
\hline 10. & $\begin{array}{l}\text { Control (without treat- } \\
\text { ment) }\end{array}$ & $\begin{array}{c}26.80 \\
(20.33)\end{array}$ & $\begin{array}{c}28.44 \\
(22.68)\end{array}$ & $\begin{array}{l}27.62 \\
(21.5)\end{array}$ & $\begin{array}{c}20.09 \\
(11.80)\end{array}$ & $\begin{array}{c}19.77 \\
(11.44)\end{array}$ & $\begin{array}{c}19.93 \\
(11.62)\end{array}$ & $\begin{array}{l}55.47 \\
(67.87)\end{array}$ & $\begin{array}{c}54.26 \\
(65.88)\end{array}$ & $\begin{array}{c}54.87 \\
(66.88)\end{array}$ & - \\
\hline \multicolumn{2}{|c|}{ SEm \pm} & 0.74 & 0.78 & 0.48 & 0.89 & 0.89 & 0.55 & 1.82 & 1.85 & 1.14 & - \\
\hline \multicolumn{2}{|c|}{$\operatorname{CD}(p=0.05)$} & 2.20 & 2.32 & 1.37 & 2.64 & 2.65 & 1.60 & 5.41 & 5.49 & 3.30 & - \\
\hline \multicolumn{2}{|c|}{ C.V. \% } & 6.00 & 6.33 & 6.16 & 8.95 & 9.17 & 9.06 & 5.09 & 5.15 & 5.12 & - \\
\hline
\end{tabular}

Data outside the paranthesis are ARCSIN transformed data 


\begin{tabular}{|c|c|c|c|c|c|c|c|c|c|c|c|c|c|}
\hline \multirow{3}{*}{$\begin{array}{l}\text { Sr. } \\
\text { No. }\end{array}$} & \multirow[t]{3}{*}{ Treatment } & \multicolumn{6}{|c|}{ Growth parameters (45 DAS) } & \multicolumn{6}{|c|}{ Yield parameters (At harvest) } \\
\hline & & \multicolumn{2}{|c|}{$\begin{array}{l}\text { Av. plant } \\
\text { height }\end{array}$} & \multicolumn{2}{|c|}{$\begin{array}{l}\text { Av. main root } \\
\text { length }\end{array}$} & \multicolumn{2}{|c|}{$\begin{array}{l}\text { Av. no. of root } \\
\text { nodules/plant }\end{array}$} & \multicolumn{2}{|c|}{$\begin{array}{l}\text { Av. no. of } \\
\text { pods/plant }\end{array}$} & \multicolumn{2}{|c|}{$\begin{array}{l}\text { Av. no. of } \\
\text { seeds /pod }\end{array}$} & \multicolumn{2}{|c|}{$\begin{array}{c}\text { Av. test } \\
\text { weight (1000 } \\
\text { seed weight) }\end{array}$} \\
\hline & & $\mathrm{Cm}$ & $\begin{array}{l}\text { In- } \\
\text { crease } \\
(\%)\end{array}$ & $\mathrm{Cm}$ & $\begin{array}{l}\text { In- } \\
\text { crease } \\
(\%)\end{array}$ & $\begin{array}{l}\text { Num- } \\
\text { ber }\end{array}$ & $\begin{array}{c}\text { Increase/ } \\
\text { Decrease } \\
(\%)\end{array}$ & $\begin{array}{c}\text { Num- } \\
\text { ber }\end{array}$ & $\begin{array}{l}\text { In- } \\
\text { crease } \\
(\%)\end{array}$ & $\begin{array}{l}\text { Num- } \\
\text { ber }\end{array}$ & $\begin{array}{l}\text { In- } \\
\text { crease } \\
(\%)\end{array}$ & gm & $\begin{array}{l}\text { I } n- \\
\text { crease } \\
(\%)\end{array}$ \\
\hline 1. & $\begin{array}{l}\text { Carbendazim } \\
\text { (Bavistin 50\% } \\
\text { WP) }\end{array}$ & 39.67 & 40.52 & 16.88 & 31.57 & 11.68 & 11.77 & 11.90 & 15.09 & 7.31 & 15.12 & 41.30 & 15.04 \\
\hline 2. & $\begin{array}{l}\text { Kasugomycin } \\
\text { (Kasugomycin } \\
12 \% \text { WP) }\end{array}$ & 31.67 & 12.19 & 12.89 & 0.47 & 10.35 & -0.96 & 10.47 & 1.26 & 6.43 & 1.26 & 36.35 & 1.25 \\
\hline 3. & $\begin{array}{l}\text { Thiram (Thi- } \\
\text { rum } 75 \% \text { WP) }\end{array}$ & 35.89 & 27.13 & 15.50 & 20.81 & 11.71 & 12.06 & 11.58 & 11.99 & 7.12 & 12.13 & 40.21 & 12.01 \\
\hline 4. & $\begin{array}{l}\text { Captan (Cap- } \\
\tan 75 \% \text { WP) }\end{array}$ & 37.00 & 31.07 & 16.38 & 27.67 & 11.59 & 10.91 & 11.85 & 14.60 & 7.28 & 14.65 & 41.14 & 14.60 \\
\hline 5. & $\begin{array}{l}\text { Carbendazim } \\
+ \text { mancozeb } \\
\text { (Sixer } 75 \% \text { WP) }\end{array}$ & 41.53 & 47.11 & 17.20 & 34.06 & 10.50 & 0.48 & 12.33 & 19.25 & 7.58 & 19.37 & 42.81 & 19.25 \\
\hline 6. & $\begin{array}{l}\text { MEMC (Emis- } \\
\text { an 6\% WP) }\end{array}$ & 33.55 & 18.85 & 14.96 & 16.60 & 10.23 & -2.11 & 10.67 & 3.19 & 6.55 & 3.15 & 37.01 & 3.09 \\
\hline 7. & $\begin{array}{l}\text { Mancozeb } \backslash(\mathrm{Di}- \\
\text { athane M-45, } \\
75 \% \text { WP) }\end{array}$ & 32.66 & 15.69 & 13.93 & 8.57 & 11.36 & 8.71 & 10.66 & 3.09 & 6.55 & 3.15 & 37.00 & 3.06 \\
\hline 8. & $\begin{array}{l}\text { Thiophanate } \\
\text { methyl } \\
\text { (Topsin M- } \\
75 \% \text { WP) }\end{array}$ & 39.91 & 41.37 & 17.07 & 33.05 & 10.50 & 0.48 & 12.17 & 17.70 & 7.48 & 17.80 & 42.26 & 17.72 \\
\hline 9. & $\begin{array}{l}\text { Difenconazole } \\
\text { (Score } 25 \% \text { EC) }\end{array}$ & 35.73 & 26.57 & 15.10 & 17.69 & 10.71 & 2.49 & 11.09 & 7.25 & 6.82 & 7.40 & 38.51 & 7.27 \\
\hline 10. & $\begin{array}{l}\text { Control (with- } \\
\text { out treatment) }\end{array}$ & 28.23 & - & 12.83 & - & 10.45 & - & 10.34 & - & 6.35 & - & 35.90 & - \\
\hline \multicolumn{2}{|c|}{ SEm \pm} & 1.74 & - & 0.60 & - & 0.37 & - & 0.48 & - & 0.25 & - & 1.38 & - \\
\hline \multicolumn{2}{|c|}{$\mathrm{CD}(p=0.05)$} & 5.16 & - & 1.77 & - & 1.09 & - & 1.41 & - & 0.73 & - & 4.09 & - \\
\hline \multicolumn{2}{|c|}{ C.V. \% } & 8.46 & - & 6.75 & - & 5.92 & - & 7.28 & - & 6.16 & - & 6.07 & - \\
\hline
\end{tabular}

carbendazim $(40.52 \%)$, captan $(31.07 \%)$, thiram $(27.13 \%)$, difenconazole (26.57\%), MEMC (18.85\%), mancozeb (15.69\%) and kasugamycin (12.19\%).

\subsubsection{Main root length}

Average main root length was significantly increased in all the treatments over the control. It was maximum in dry seed treatment with carbendazim+mancozeb $(17.20 \mathrm{~cm})$ which was statistically at par with thiophanate methyl $(17.07 \mathrm{~cm})$, carbendazim $(16.88 \mathrm{~cm})$ and captan $(16.38 \mathrm{~cm})$. Next best treatment in order to merit was thiram $(15.50 \mathrm{~cm})$ followed by difenconazole $(15.10 \mathrm{~cm}), \operatorname{MEMC}(14.96 \mathrm{~cm})$, mancozeb
$(13.93 \mathrm{~cm})$ and kasugamycin $(12.89 \mathrm{~cm})$. Average main root length was recorded the lowest in the control $(12.83 \mathrm{~cm})$. Maximum increase in main root length was recorded in carbendazim+mancozeb (34.06\%) followed by thiophanate methyl (33.05\%), carbendazim (31.57\%), captan $(27.67 \%)$, thiram $(20.81 \%)$, difenconazole (17.69\%), MEMC (16.60\%), mancozeb $(8.57 \%)$ and kasugamycin $(0.47 \%)$.

3.4.3. Numbers of root nodules plant ${ }^{-1}$

Average numbers of root nodules plant ${ }^{-1}$ was significantly increased in some of the treatments over the control. It was higher in dry seed treatment with thiram (11.71) but was 


\begin{tabular}{|c|c|c|c|c|c|}
\hline \multirow{2}{*}{$\begin{array}{l}\text { Sr. } \\
\text { No. }\end{array}$} & \multirow[t]{2}{*}{ Treatment } & \multicolumn{4}{|c|}{ Yield $\left(\mathrm{Kg} \mathrm{ha}^{-1}\right)$} \\
\hline & & $\begin{array}{l}\text { First } \\
\text { year }\end{array}$ & $\begin{array}{l}\text { second } \\
\text { year }\end{array}$ & Pooled & $\begin{array}{c}\text { Increase } \\
(\%)\end{array}$ \\
\hline 1. & $\begin{array}{l}\text { Carbendazim } \\
\text { (Bavistin 50\% } \\
\text { WP) }\end{array}$ & 785.78 & 756.92 & 771.35 & 42.75 \\
\hline 2. & $\begin{array}{l}\text { Kasugomycin } \\
\text { (Kasugomycin } \\
12 \% \text { WP) }\end{array}$ & 626.71 & 630.52 & 628.61 & 16.34 \\
\hline 3. & $\begin{array}{l}\text { Thiram (Thi- } \\
\text { rum } 75 \% \text { WP) }\end{array}$ & 728.46 & 723.63 & 726.04 & 34.37 \\
\hline 4. & $\begin{array}{l}\text { Captan (Cap- } \\
\tan 75 \% \text { WP) }\end{array}$ & 762.65 & 726.37 & 744.51 & 37.79 \\
\hline 5. & $\begin{array}{l}\text { Carbendazim } \\
\text { +mancozeb } \\
\text { (Sixer } 75 \% \text { WP) }\end{array}$ & 819.06 & 817.74 & 818.40 & 51.46 \\
\hline 6. & $\begin{array}{l}\text { MEMC (Emisan } \\
6 \% \text { WP) }\end{array}$ & 644.85 & 642.25 & 643.55 & 19.10 \\
\hline 7. & $\begin{array}{l}\text { Mancozeb } \backslash(\text { Di- } \\
\text { athane M-45, } \\
75 \% \text { WP) }\end{array}$ & 646.55 & 643.91 & 645.23 & 19.41 \\
\hline 8. & $\begin{array}{l}\text { Thiophanate } \\
\text { methyl } \\
\text { (Topsin M- } \\
75 \% \text { WP) }\end{array}$ & 807.38 & 774.08 & 790.73 & 46.34 \\
\hline 9. & $\begin{array}{l}\text { Difenconazole } \\
\text { (Score 25\% EC) }\end{array}$ & 678.44 & 645.31 & 661.87 & 22.49 \\
\hline 10. & $\begin{array}{l}\text { Control (with- } \\
\text { out treatment) }\end{array}$ & 547.24 & 533.44 & 540.34 & - \\
\hline \multicolumn{2}{|c|}{ SEm \pm} & 30.45 & 32.68 & 19.68 & - \\
\hline \multicolumn{2}{|c|}{$\mathrm{CD}(p=0.05)$} & 90.48 & 97.09 & 56.99 & - \\
\hline \multicolumn{2}{|c|}{ C.V. $\%$} & 7.48 & 8.21 & 7.85 & - \\
\hline
\end{tabular}

Data outside the paranthesis are ARCSIN transformed data

statistically at par with carbendazim (11.68), captan (11.59), difenconazole, (11.36) and mancozeb (10.71). Next best treatment in order to merit was carbendazim + mancozeb (10.50) followed by thiophanate methyl (10.50) as compared to the control (10.45). It was significantly decreased in MEMC (10.23) followed by kasugamycin (10.35) over the control. Maximum increase in numbers of root nodules plant ${ }^{-1}$ was recorded in thiram (12.06\%) followed by carbendazim (11.77\%), captan (10.91\%), mancozeb (8.71\%), difenconazole (2.49\%), carbendazim + mancozeb $(0.48 \%)$ and thiophanate methyl $(0.48 \%)$, while maximum decrease in numbers of root nodules plant ${ }^{-1}$ was recorded in MEMC $(2.11 \%)$ followed by kasugamycin $(0.96 \%)$ over the control.

\subsubsection{Yield parameters}

\subsubsection{Numbers of pods plant ${ }^{-1}$}

Average numbers of pods plant ${ }^{-1}$ was significantly increased in all the treatments over the control. It was maximum in dry seed treatment with carbendazim + mancozeb (12.33) but was statistically at par with thiophanate methyl (12.17), carbendazim (11.90), captan (11.85), thiram (11.58) and difenconazole (11.09). Next best in order to merit was MEMC (10.67) followed by mancozeb (10.66) and kasugamycin (10.47). Average numbers of pods plant ${ }^{-1}$ was recorded the lowest in the control (10.34). Maximum increase in numbers of pods plant ${ }^{-1}$ was recorded in carbendazim + mancozeb (19.25\%) followed by thiophanate methyl (17.70\%), carbendazim(15.09\%), captan (14.60\%), thiram (11.99\%), difenconazole (7.25\%), MEMC (3.19\%), mancozeb (3.09\%) and kasugomycin (1.26\%).

\subsubsection{Numbers of seeds pod ${ }^{-1}$}

Average numbers of seeds pod ${ }^{-1}$ was significantly increased in all the treatments over the control. It was maximum in dry seed treatment with carbendazim + mancozeb (7.58) which was statistically at par with thiophanate methyl (7.48), carbendazim (7.31), captan (7.28) and thiram (7.12). Next best treatment in order to merit was difenconazole (6.82) followed by MEMC (6.55), mancozeb (6.55) and kasugamycin (6.43). Average numbers of seeds pod ${ }^{-1}$ was recorded the lowest in the control (6.35). Maximum increase in numbers of seeds pod $^{-1}$ was recorded in carbendazim+mancozeb (19.37\%) followed by thiophanate methyl $(17.80 \%)$, carbendazim (15.12\%), captan (14.65\%), thiram (12.13\%), difenconazole (7.40\%), MEMC (3.15\%), mancozeb (3.15\%) and kasugamycin (1.26\%).

\subsubsection{Test weight}

Average test weight was significantly increased in all the treatments over the control. It was maximum in dry seed treatment with carbendazim + mancozeb $(42.81 \mathrm{~g})$ but was statistically at par with thiophanate methyl (42.26 g), carbendazim (41.30 g), captan (41.14 g) and thiram (40.21 g). Next best treatment in order to merit was difenconazole (38.51 g) followed by MEMC (37.01 g), mancozeb (37.00 g) and kasugamycin $(36.35 \mathrm{~g})$. Average test weight was recorded lowest in the control $(35.90 \mathrm{~g})$. Maximum increase in test weight was recorded in carbendazim + mancozeb (19.25\%) followed by thiophanate methyl (17.72\%), carbendazim (15.04\%), captan (14.60\%), thiram (12.01\%), difenconazole (7.27\%), MEMC (3.09\%), mancozeb (3.06\%) and kasugamycin (1.25\%).

\subsubsection{Yield}

The pooled data of two years indicated that grain yield (ha$\left.{ }^{1}\right)$ was significantly increased in all the treatments over the control. It was significantly higher in dry seed treatment with carbendazim + mancozeb $\left(818.40 \mathrm{~kg} \mathrm{ha}^{-1}\right)$ as compared to the rest but was statistically at par with thiophanate methyl (790.73 $\left.\mathrm{kg} \mathrm{ha}^{-1}\right)$, and carbendazim (771.35 kg ha-1). Next 
best treatment in order to merit was captan (744.51 $\left.\mathrm{kg} \mathrm{ha}^{-1}\right)$ followed by thiram (726.04 kg ha-1). Comparatively poor yield was recorded in difenconazole (661.87 $\left.\mathrm{kg} \mathrm{ha}^{-1}\right)$, MEMC (645.23 $\left.\mathrm{kg} \mathrm{ha}^{-1}\right)$, mancozeb (643.55 $\left.\mathrm{kg} \mathrm{ha}^{-1}\right)$ and kasugamycin (628.61 $\left.\mathrm{kg} \mathrm{ha}^{-1}\right)$. Lowest yield was recorded in the control $(540.34 \mathrm{~kg}$ $\left.\mathrm{ha}^{-1}\right)$. Maximum increase in grain yield was recorded in mixed formulation carbendazim + mancozeb (51.46\%) followed by thiophanate methyl (46.34\%), carbendazim (42.75\%), captan (37.79\%), thiram (34.37\%), difenconazole (22.19\%), MEMC (19.41\%), mancozeb (19.10\%) and kasugamycin (16.34\%).

The present results are more or less similar with the earlier workers. Fungicidal seed treatment with Agallol, Captan or Thirum (Suhag, 1975) and Bavistin, Thiram and Diathane M-45 (Zote and Mayee, 1982) proved better in promoting the germination and seedling vigour of moong beanseeds. Reddy et al. (1992) showed that fungicidal seed dressing with flutolanil, thiophanate-methyl or carbendazim effectively controlled damping-off of Vigna radiata, while Mancozeb and zineb were the least effective. Moreover to these results, Dash and Narain (1996) also found that pre-treatment of seeds of $V$. radiata with carbendazim + thiram followed by Thiram, Brassicol [quintozene], Difolatan [captafol], Dithane M-45 (flowable) [mancozeb] and Vitavax [carboxin] eliminated seed mycoflora and improved seed germination considerably for most of the test crops. Agrawal (1989) noticed that seed treatment with carbendazim reduced seed and seedling mortality caused by $M$. phaseolina in V. radiata. The initial leaf blight phase of the disease was also found less. Bidari et al. (1992) observed that seed treatment with Bavistin [carbendazim] gave the best control of seed borne pathogens by reducing seed rot, seedling mortality and resulting in the highest yield of the $V$. radiata crop. Good control was also obtained in Parasan [Phenyl mercury acetate], Dithane M-45 [mancozeb] and Thiram at $2 \mathrm{~g} \mathrm{~kg}^{-1}$ seed under field condition. Khan and Khan (2006) also reported that seed treatment with $0.10 \%$ Topsin $\mathrm{M}$ showed significantly higher reduction in Macrophomina leaf spot incidence at 45, 55 and 70 days after sowing and recorded the highest increase in green gram yield (46.8\%) followed by benomyl (32.9\%) and carbendazim (31.40\%). The control of anthracnose and Alternaria leaf spot by fungicidal seed treatment carried out here is the first exempt but the work done in other crops was in similar line with the present study. Suradkar (2010) found that maximum control of anthracnose caused by $C$. capsici was obtained in seed treatment of carbendazim ( $81.57 \%$ ) followed by thiram (76.31\%), MEMC (64.47\%) and mancozeb (63.15\%) along with better increased in seed germination in blackgram. Laxminarayanarao (2006) found that seed dressing with carbendazim + iprodione was most effective in eliminating seed borne infections of Alternaria sp. causing Alternaria blight in sunflower. The results of fungicidal seed treatment on root nodulation are more or less similar with the earlier workers in green gram and other crops. Patel et al. (1989) found that Dithane M-45 and Bavistin had no adverse effect on rhizobia isolated from green gram in in vitro. No adverse effect in root nodulation due to dry seed treatment with captan and thiram in soyabean (Nene et al., 1969), thiram in chickpea (Welty et al., 1988) were reported. Moreover, Vyas et al. (1990) concluded that soyabean seeds treated with thiram do not make any difference in nodule number and nodule dry weight over non treated seeds. Ehteshamul-Haque and Ghaffar, (1995) found that seed treatment with Bavistin was not only control root infecting fungi but also produce better nodulation in soybean. The earlier results of Lennox and Alexander (1981) gave the confirmation and possible reasons of the present results in a very clear way who noticed that naturally tolerant Rhizobial strains showed compatibility with fungicides and resulted in increase in nodulation and better protection from invasion by root infecting fungi in Phaseolus vulgaris.

\section{Conclusion}

Dry seed treatment with carbendazim+mancozeb or thiophanate methyl or carbendazim @ $2.5 \mathrm{~g} \mathrm{~kg}^{-1}$ of seeds was recommended in green gram to overcome pre and post emergence mortality and to get better seed germination, plant height, root length, numbers of root nodules plant ${ }^{-1}$, number of pods, number of seeds pod ${ }^{-1}$, average test weight and yield.

\section{References}

Agrawal, S.C., 1989. Diseases of green gram and black gram. International Book distributors, 1-2, 5, 28-29, 32-33, 159, 181, 269.

Bidari, V.B., Dayanand, M., Anahosur, K.H., Mannur, D.M., 1992. Field evaluation of seed treating fungicides against seed rot in green gram. Indian Journal of Pulses Research 5, 94-96.

Bilgrami, K.S., Sinha, R.K., Prasad, T., 1978. Effect of fungal flora on the seed contents of moong. Indian Phytopathology 31, 476-479.

Dash, S.K., Narain, A., 1996. Efficacy of selected fungicides on seed-borne fungi and on percentage of germination of diseased seeds of crops. Crop Research- Hisar 11, 207-211.

Ehteshamul-Haque, S., Ghaffar, A., 1995. Effect of Bradirhizobium japonicum and fungicides in the control of root rot disease of soybean. Pakistan Journal of Botany 27, 227-232.

John, M.P., 1991. The mung bean. Oxford and IBH Publishing Co. Pvt. Ltd. 66 Janpath, New Delhi, 126-127.

Khan, A.A., Khan, R.U., 2006. Management of macrophomina leaf spot of Vigna radiata by fungicides. Annals-of-PlantProtection-Sciences 14, 258-259.

Laxminarayanarao, M.S., 2006. Studies on seed-borne fungal diseases of sunflower and their management with special reference to the alternaria blight. Ph.D. Thesis submitted to the University of Agricultural Sciences, Dharwad. 
Lennox, L.B., Alexander, M., 1981. Fungicide enhancement of nitrogen fixation and colonization of Phaseolus vulgaris by Rhizobium phaseoli. Applied and Environmental Microbiology 41, 404-411.

Nene, Y.L., Agarwal, K., Srivastava, S.S.L., 1969. Influence of fungicidal seed treatment on the emergence and nodulation of soybean. Pesticides 3, 26-27.

Patel, G.Z., Lodha, P.C., Patel, I.S., 1989). In vitro effect of antibiotics and fungicides on Rhizobium sp., isolated from green gram. Pestology 13, 28-30.

Rangaswami, G., Prasad, N., 1960. A new seedling blight of Phaseolusaureus Roxb. And Phaseolus mungo L. Indian Phytopathology 12, 184-185.

Rayen, G.F., 1961. Seedling albinism induced by an extract of Alternaria tenuis. Science 134, 833-834.

Reddy, K.S., Rao, K.C., Reddy, M.S., 1992. Evaluation of some new fungicides against Rhizoctonia solani Kuhn, the incitant of damping-off of mungbean. Indian Journal of Plant Protection 20, 37-42.

Sinha, R.K., Prasad, T., 1981. Effect of fungal metabolites on seed germination, mycobial association and seedling growth of Mung. Indian Phytopathology 34, 515-517.
Suhag, L.S., 1975. Fungal flora of Moong (Phaseolus aureus) seeds: pathology and control. Indian Journal of Mycology and Plant Pathology 5, 165-168.

Suradkar, S.R., 2010. Investigation on anthracnose [Colletotrichum capsici (Syd.) Butler and Bisby] of Blackgram (Vigna mungo L. Hepper) under south Gujarat condition. M.Sc. (Agri.) thesis submitted to NAU, Navsari.

Vyas, S.C., Vyas, A., Mahajan, K.C., Shroff, V.N., 1990. Effect of fungicides on mycorrhizal and rhizobial development in soybean. Current trend in Mycorrhizal Research. Proceedings on the Mycorrhizae at Haryana Agricultural University, Hissar, India, 188-189.

Welty, L.E., Prestbye, L.S., Hall, J.A., Mathre, D.E., Ditterline, R.L., 1988. Effect of fungicide seed treatment and rhizobia inoculation on chickpea production. Applied Agricultural Research 3, 17-20.

Zote, K.K., Mayee, C.D., 1982. Influence of fungicidal seed treatment during storage on seed borne fungi of mung bean. Pesticides, 10-12. 